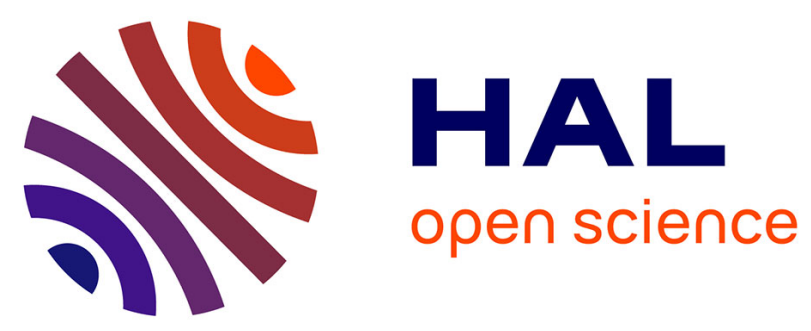

\title{
Circularly Polarized Leaky-Wave Antenna Based on a Dual-Mode Hollow Waveguide
}

\author{
A. Dorle, R. Gillard, E. Menargues, M. van Der Vorst, E. de Rijk, P. \\ Martin-Iglesias, M. García-Vigueras
}

\section{- To cite this version:}

A. Dorle, R. Gillard, E. Menargues, M. van Der Vorst, E. de Rijk, et al.. Circularly Polarized Leaky-Wave Antenna Based on a Dual-Mode Hollow Waveguide. IEEE Transactions on Antennas and Propagation, 2021, 69 (9), pp.6010-6015. 10.1109/TAP.2021.3083783 . hal-03367511

\section{HAL Id: hal-03367511 \\ https://hal.science/hal-03367511}

Submitted on 15 Oct 2021

HAL is a multi-disciplinary open access archive for the deposit and dissemination of scientific research documents, whether they are published or not. The documents may come from teaching and research institutions in France or abroad, or from public or private research centers.
L'archive ouverte pluridisciplinaire HAL, est destinée au dépôt et à la diffusion de documents scientifiques de niveau recherche, publiés ou non, émanant des établissements d'enseignement et de recherche français ou étrangers, des laboratoires publics ou privés. 


\title{
Circularly-Polarized Leaky-Wave Antenna Based on a Dual-Mode Hollow Waveguide
}

\author{
A. Dorlé, R. Gillard, E. Menargues, M. Van Der Vorst, E. De Rijk, P. Martín-Iglesias and M. García-Vigueras
}

\begin{abstract}
This paper presents a compact leaky-wave antenna providing circular polarization from a dual-mode hollow waveguide. The monolithic structure is based on a rectangular metallic guide that is perforated with crossed slots. The antenna is fed by a standard WR42 port, which is connected to the leaky waveguide by a transition that allows for dual-mode excitation. The axial ratio is determined by the size of the waveguide section and the dimensions of the crosses. In order to preserve the polarization purity, the geometry of the first radiating elements is modulated. Designed at K-band, the present proposal is suitable for space applications since it relies on a full-metal structure. A design methodology is proposed based on dual-mode dispersion analysis. Selective laser melting is used to monolithically 3D-print the antenna. Measurements are in good agreement with simulations, and the axial ratio is $0.92 \mathrm{~dB}$ at $20.5 \mathrm{GHz}$.
\end{abstract}

Index Terms - Additive manufacturing, circular polarization, slotted waveguide, dual-mode, K-band, leaky waves, selective laser melting.

\section{INTRODUCTION}

Circular polarization (CP) is often required for space applications, being implemented for telecom links between satellites and base stations, end-users on Earth, or landers for deep space missions [1]. $\mathrm{CP}$ is also used for potential future inter-satellite links (ISLs) within constellations of small satellites [2]. In addition to CP, the space environment also demands low loss components and to avoid the use of dielectric materials that are directly exposed to free space [3]. As a result, structures based on metallic waveguides (WGs) are typically preferred in this context. Arrays of horns are the most commonly employed solutions, providing robust performance and wideband operation at the cost of relying on cumbersome WG beamforming networks. Promising alternatives presenting higher compactness are based on leaky-wave antennas (LWAs) and slotted arrays, whose profile is significantly lower since they avoid the need for complex feeding networks [4]. The trade-off in this case concerns the bandwidth of operation, which is narrower [5], [6]-[7].

Two main techniques can be identified in the literature to conceive compact CP antennas based on the radiation from leaky waves (LWs) on hollow WGs. On the one hand, single linearly-polarized slotted arrays can be combined with external polarizers [5]. However, the co-design of the polarizer and the slotted array is not obvious and needs iterative steps to find the best solution. Besides, such compound architectures require further assembly steps and may result in costly and bulky prototypes. On the other hand, a second technique can be found using CP-polarized LW radiating elements,

Manuscript submitted March 16, 2020. This work was supported in part by the European Space Agency under the grant NPI 486-2016, Rennes Métropole for the funds AIS 17C0481.

A. Dorlé, M. García-Vigueras and R. Gillard are with IETR, UMR CNRS 6164, INSA Rennes, 35708 Rennes, France (e-mail: aurelie.dorle@insarennes.fr, maria.garcia-vigueras@insa-rennes.fr).

P. Martín-Iglesias and M. Van Der Vorst are with the European Space Agency (ESA), ESTEC, Noordwijk, The Netherlands.

E. Menargues and E. De Rijk are with SWISSto12, CH-1020 Renens, Switzerland (e-mail: e.menargues@swissto12.ch).

Color versions of one or more of the figures in this communication are available online at http://ieeexplore.ieee.org.

Digital Object Identifier 10.1109/TAP.2016.xxx thus avoiding the need for external polarizers. The LWA presented in [6] has the advantage to be simple to design and manufacture, but its monomodal structure and asymmetric aperture do not allow dualpolarization. Furthermore, the cross-pol levels attained in K- and Kabands remain above $-20 \mathrm{~dB}$. Other authors have proposed to generate $\mathrm{CP}$ from symmetric LWAs basing on dual-mode operation [7]. In this case, the antenna has a compound feeding network consisting of a magic- $T$, phase shifters and attenuators. This assembly is needed to tune the LWA axial ratio (AR) by controlling the amplitude and phase of both modes at the antenna input.

This article presents a LWA that induces CP from two degenerated modes together with a design methodology based on dual-mode dispersion analysis. Such an original methodology allows understanding the LW nature of the antenna and synthesizing CP while using a very simple transition (in contrast to the bulky network needed in [7]. In particular, the propagating modes $\mathrm{TE}_{01}$ and $\mathrm{TE}_{10}$, are excited simultaneously at the input of the LWA with same power and phase. Single CP is achieved by designing properly the dimensions of the WG section and the aperture perforations. The structure can be interpreted as a combination of transversely slotted waveguide and longitudinally slotted waveguide LWAs. This proposal evolves from a previous contribution from these authors that concerned the generation of dual-linear polarization and sidelobe-level reduction [8], [9]. It should be noted that we restrict here to the case of single-CP, but extension to dual-CP is straightforward by only adding an orthomode transducer (OMT). This easy extension is not possible in other similar structures [6].

In order to validate the antenna operation principle and the design methodology, a prototype is conceived at K-band. The LWA has been monolithically manufactured using a metal Additive Manufacturing (AM) process based on Selective Laser Melting (SLM) combined with metal plating. Previous work by these authors considered a different technique based on polymer 3D-printing [8], [9]. To their best knowledge, this is the first proof of successful SLM prototyping of a leaky-waveguide. Moreover, the enhancement of the antenna radiation efficiency is proven here for the first time through the post-processing of the piece by metal plating.

Section II introduces the antenna structure and the study of the modes dispersion. Section III presents the design process to generate CP. Section IV discusses the employed AM manufacturing technique. Section V shows the antenna measured performance. Good agreement is observed compared with full wave simulations, thus validating the proposed methodology based on the control of the LW propagation constants. Finally, conclusions are given in Section VI.

\section{Dual-Mode LWA: Structure AND Dispersion ANALysis}

The structure of the proposed LWA is shown in Fig. 1. The radiating aperture is presented in Fig. 1 (a) and is created on the top wall of a rectangular and hollow WG of length $L_{A}$. The aperture is composed of periodically perforated crossed slots centered with respect to the WG lateral walls. The two arms in a cross may be of 


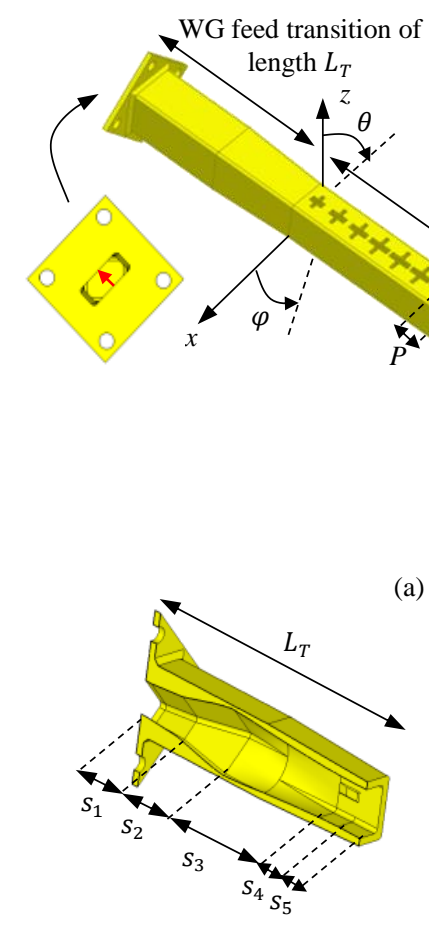

(b) (a)

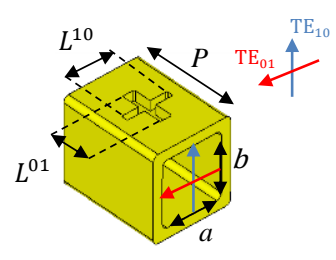

(c)
Fig. 1. Topology of the proposed LWA. (a) Full structure, (b) Single-to-dual mode WG feed transition, (c) Unit cell. Dimensions for the K-band design are: $P=7 \mathrm{~mm}$, metal thickness $=1.4 \mathrm{~mm}, a=9 \mathrm{~mm}, b=8.8 \mathrm{~mm}, L^{01}=$ $6.2 \mathrm{~mm}, L^{10}=7.3 \mathrm{~mm}, L_{A}=136 \mathrm{~mm}, s_{1}=7.9 \mathrm{~mm}, s_{2}=11.8 \mathrm{~mm}$, $s_{3}=23.7 \mathrm{~mm}, s_{4}=4.6 \mathrm{~mm}, s_{5}=5.5 \mathrm{~mm}$. The widths of the cross arms are $1 \mathrm{~mm}$ along $x$, and $1.76 \mathrm{~mm}$ along $y$.

different length, and their dimensions may vary along the $y$-axis [8]. The LWA is fed through a WR42 waveguide. As it is evidenced in Fig. 1 (a) and (b), a WG feed transition of length $L_{T}$ couples the energy from the input port to the $\mathrm{TE}_{10}$ and $\mathrm{TE}_{01}$ modes of the hollow WG [10]. The precise design of this transition is explained in Section III. Finally, the LWA is closed at its end by a metal plate, operating as a short circuit.

The basic underlying principle of the considered dual-mode LWA is explained in [7], [8] and [9]. In brief, the degenerated $\mathrm{TE}_{10}$ and $\mathrm{TE}_{01}$ modes excite two LWs which propagate along the $y$-axis with respective complex wavenumbers: $k_{y}^{10 / 01}=\beta^{10 / 01}-j \alpha^{10 / 01}$ [4]. The leakage rates $\alpha^{10 / 01}$ express the amount of energy leaked through the slots, thus determining the magnitude of the antenna aperture illumination for each polarization. The phase constants $\beta^{10 / 01}$ govern the antenna pointing angle $\theta_{R A D}^{10 / 01}$ for each polarization. Independent control of $\beta^{10 / 01}(y)$ and $\alpha^{10 / 01}(y)$ is possible at a fixed frequency in order to taper the antenna illumination [8].

\section{A. Radiation from $T E_{01}$ mode}

When the leaky hollow WG is excited by the $\mathrm{TE}_{01}$ mode, the near field at $z=b$ may be derived from classical WG theory [11].

$$
H_{y}^{01}=-A^{01} e^{-j k_{y}^{01} y}
$$

It is hence evidenced that the slotted aperture is excited by a single $H_{y}^{01}$ component. Equivalence principle then allows for expressing the final radiating current (2) [12]. A complex coefficient $c^{01}$ is added in the expression, representing the coupling between the guided and radiating currents. The current has a single $x$ component that interacts with the cross arm along $y$ (see Fig. 1(c)).

$$
\overrightarrow{J_{S}^{01}}=\overrightarrow{e_{z}} \times c^{01} \vec{H}=-c^{01} H_{y}^{01} \overrightarrow{e_{x}}=c^{01} A^{01} e^{-j k_{y}^{01} y} \overrightarrow{e_{x}}
$$

The resulting far field is found by integrating the current (2) [12]. By evaluating it in the plane of interest $\varphi=\pi / 2$, it is theoretically confirmed that the $\mathrm{TE}_{01} \mathrm{LW}$ generates a $\varphi$-polarization:

$$
\left.E_{\varphi}^{01}\right|_{\varphi=\frac{\pi}{2}}=c^{01} A^{01} w_{S}^{01} \frac{\eta k_{0} e^{-j k_{0} r}}{4 \pi r} \frac{e^{j L_{A}\left(k_{0} \sin \theta-k_{y}^{01}\right)}-1}{k_{0} \sin \theta-k_{y}^{01}} e^{j k_{0} b \cos \theta}
$$

\section{B. Radiation from $T E_{10}$ mode}

A similar procedure may be followed to express the far field generated by the $\mathrm{TE}_{10} \mathrm{LW}$. The corresponding near field propagating at the top of the leaky hollow $\mathrm{WG}$, at $z=b$, is given in (4) and (5), where $k_{c}^{10}=\sqrt{k_{0}^{2}-\left(\beta^{10}\right)^{2}}$.

$$
\begin{gathered}
H_{x}^{10}=\frac{j k_{y}^{10} \pi}{\left(k_{c}^{10}\right)^{2} a} A^{10} \sin \left(\frac{\pi x}{a}\right) e^{-j k_{y}^{10} y} \\
H_{y}^{10}=A^{10} \cos \left(\frac{\pi x}{a}\right) e^{-j k_{y}^{10} y}
\end{gathered}
$$

Contrary to the previous $\mathrm{TE}_{01} \mathrm{LW}$, two components excite now the slotted aperture: $H_{x}^{10}$ and $H_{y}^{10}$ (as $E_{z}^{10}$ is orthogonal to the surface). The radiating currents, being either $x$ - or $y$-oriented, are derived straightforwardly.

The far field radiated by the $\mathrm{TE}_{10} \mathrm{LW}$ in the $Z Y$-plane is a pure $\theta$ polarization, expressed in (6), which is provided by the symmetry of the perforations with respect to the lateral walls.

$$
\begin{aligned}
& \left.E_{\theta}^{10}\right|_{\varphi=\frac{\pi}{2}} \\
& =-j 2 c^{10} A^{10}\left(\frac{a}{\pi}\right)^{2} \cos \theta k_{y}^{10} \frac{\eta k_{0} e^{-j k_{0} r}}{4 \pi r} \frac{e^{j L_{A}\left(k_{0} \sin \theta-k_{y}^{10}\right)}-1}{k_{0} \sin \theta-k_{y}^{10}} e^{j k_{0} b \cos \theta}
\end{aligned}
$$

\section{Dual-Mode Dispersion Analysis}

The frequency dispersion of the two LWs propagating in the antenna has been computed with a full-wave solver (HFSS). From the simulated results, the following well-known expressions are used in order to compute the phase and attenuation constants: $\beta^{10 / 01}=$ $k_{0} \sin \left(\theta_{\mathrm{RAD}}^{10 / 01}\right)$ and $\alpha^{10 / 01}=-\ln \left(\left|\mathrm{S}_{21}^{10 / 01}\right|^{2} / 1-\left|\mathrm{S}_{11}^{10 / 01}\right|^{2}\right) / 2 L_{A}$. Main results are shown in Fig. 2. In each dispersion diagram, the leakage rate is represented only from the cutoff frequency, since this is the regime in which our LWA will operate. More specifically, these graphs show the impact of the perforations dimensions on the wavenumbers of both LWs when considering uniform LWAs (all the crosses are identical along the $y$-axis). Illustrative cases are considered where the lengths of the crossed-slot arms $\left(L^{10}\right.$ and $L^{01}$, see Fig. 1) vary while their widths are kept constant. The main structure dimensions are given in the caption of Fig. 2.

According to Fig. 2 (a) and (b), the two LWs phase constants exhibit an identical behavior, and it is coherent with the basic theory on perforated hollow WGs [4]. Especially, they increase from zero at the cutoff frequency, and tend asymptotically to the value $k_{0}$ as the frequency increases. The specific dimensions of the hollow WG cross section (namely, $a, b$ ) have been chosen in order to assure that only the desired degenerated modes (the $\mathrm{TE}_{10}$ and the $\mathrm{TE}_{01}$ ) propagate within the frequency band of interest. Besides, the period $P$ between two consecutive crosses is chosen to avoid the excitation of propagative higher-order Floquet harmonics below $25 \mathrm{GHz}$ [4]. Fig. 2 (a) and (b) show that $L^{10}$ and $L^{01}$ have no effect on $\beta^{01}$ and $\beta^{10}$, respectively. On the contrary, $L^{10}$ and $L^{01}$ may affect $\beta^{10}$ and $\beta^{01}$, respectively, as shown by Fig. 2 (c) and (d). Indeed, each phase constant is sensitive to the cross arm which is defining the boundary condition of the associated guided mode. 


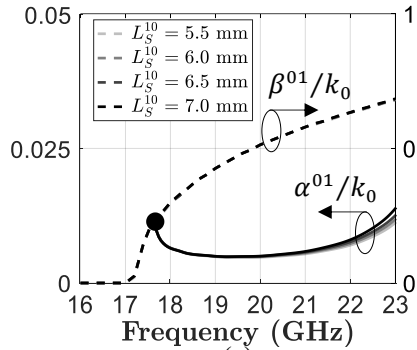

(a)

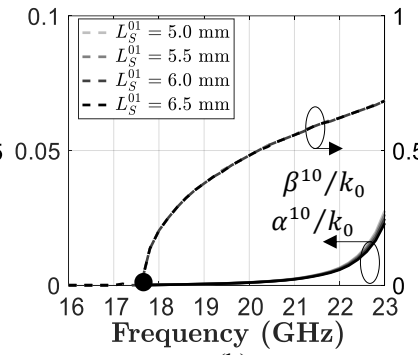

(b)

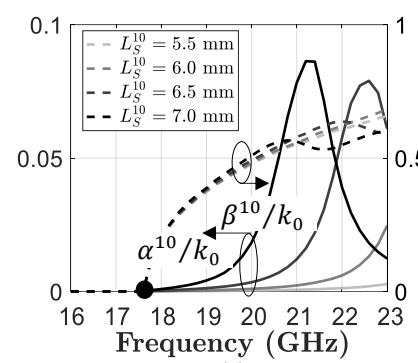

(c)

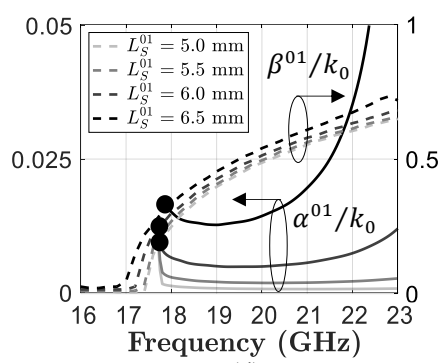

(d)

Fig. 2. Normalized phase constants (dashed lines) and leakage rates (continuous lines). Subfigures (a) and (d) show the dispersion for the perturbed TE $\mathrm{E}_{01}$ mode while (b) and (c) concern the case of the $\mathrm{TE}_{10}$. (a) and (c) present the effect of varying $L^{10}\left(L^{01}=6 \mathrm{~mm}\right)$ whereas (b) and (d) show results sweeping $L^{01}\left(L^{10}=6\right.$ $\mathrm{mm}$ ). Other dimensions of the simulated LWA are: $P=7 \mathrm{~mm}$, thickness of metal $1.4 \mathrm{~mm}, a=b=8.5 \mathrm{~mm}$, slots widths $0.7 \mathrm{~mm}$ and $L_{A}=84 \mathrm{~mm}$.

The evolution of $\alpha^{01}$ and $\alpha^{10}$ with frequency obeys quite different rules. On the one hand, Fig. 2 (a) and (d) show that $\alpha^{01}$ is greater than zero after the cutoff and then decreases smoothly as $\beta^{01}$ increases [4]. As the frequency increases, the trend is reversed, and $\alpha^{01}$ increases again to reach a maximum level (above $23 \mathrm{GHz}$ ). This evolution is related to the resonance of the crossed slot along the $y$ direction, thus to $L^{01}$. This arm determines the level of transparency experienced by the $\mathrm{TE}_{01}$ mode at the aperture, since the slot is perpendicular to $\overrightarrow{J_{S}^{01}}(2)$. This effect is further demonstrated in Fig. 2 (d), where it is clear that $L^{01}$ allows controlling $\alpha^{01}$ [8]. It should be noted that $\alpha^{01}$ is not impacted by $L^{10}$, as shown in Fig. 2 (a).

On the other hand, Fig. 2 (b) and (c) present the frequency dispersion of $\alpha^{10}$ [13]. The behavior close to the cutoff is clearly different. At the cutoff of the $\mathrm{TE}_{10}, \alpha^{10}$ is null and then increases progressively together with $\beta^{10}$. This relation is justified by the presence of the factor $k_{y}^{10}$ multiplying the $y$-oriented component of the surface current $\overrightarrow{J_{S}^{10}}$, as also seen in (6). It should be noted that such a multiplication factor does not appear in the case of $\overrightarrow{J_{S}^{01}}$. The curves in Fig. 2 (b) and (c) also show that $\alpha^{10}$ keeps on growing as the frequency increases until reaching a maximum level (which appears above $23 \mathrm{GHz}$ in the case of Fig. 2 (b)), and then decreases asymptotically towards zero. This progression was also observed in the case of $\alpha^{01}$ and is related to the resonance of the crossed slot along the $x$-direction, and therefore, to $L^{10}$. By inspecting Fig. 2 (b), it is also clear that $L^{01}$ has almost no impact on $\alpha^{10}$.

Finally, it can be concluded that the frequency dispersion associated to both radiating LWs is very similar regarding phase constants, but has significantly different trends for leakage rates. Such differences come mainly from the fact that the aperture current associated to the $\mathrm{TE}_{10}$ mode is somehow proportional to its phase constant, contrary to the one associated to the $\mathrm{TE}_{01}$ mode. At a fixed frequency, there is relatively wide margin to control independently the leakage rates associated to both radiating LWs: $\alpha^{10}$ and $\alpha^{10}$ can be tuned through $L^{10}$ and $L^{01}$ respectively. Analogous independent control is achievable for the phase constants $\beta^{10}$ and $\beta^{10}$, using the WG cross section dimensions $a$ and $b$ to modify the cutoff frequencies. Such dispersion graphs have not been presented here for the sake of conciseness [8], [9].

\section{Design Methodology for CP Antenna}

$\mathrm{CP}$ radiation can be pursued in the dual-mode LWA through the simultaneous excitation of both LWs analyzed in the previous section. To this aim, the objective is to generate and combine properly the two radiated orthogonal linear polarizations expressed in (3) and (6), which is carried out in two consecutive steps. Firstly, the perforated WG is designed, assuming equal excitation of both degenerated LWs. In coherence with the scenario considered in Section II.C., the perforated WG is assumed to be ended by a matched load in this first step. Secondly, a WG feed transition is designed to assure such dual-mode excitation from a single monomodal port. Additionally, several actions are taken to mitigate possible diffraction produced by the two LWs at the input and output of the perforated WG. These two steps are explained in the next subsections, which are followed by the performance of a design operating around $20 \mathrm{GHz}$.

\section{A. Design of Dual-Mode Perforated WG}

As explained above, the WG is here assumed to be ended by a matched load. Desired CP radiation is thus produced by the two progressive LWs on their first propagation path from $y=0$ up to $y=L_{A}$ (see Fig. 1(a)). Pure CP is theoretically achieved when $\left|E_{\theta}^{10}\right|=\left|E_{\varphi}^{01}\right|$ and $\arg \left(E_{\theta}^{10}\right)= \pm \pi / 2+\arg \left(E_{\varphi}^{01}\right)$. The analysis of (3) and (6) shows that $\mathrm{CP}$ conditions are not met by just exciting the transverse and longitudinal slots with equal orthogonal modes. Indeed, the coupling coefficients $c^{10 / 01}$ have also to be taken into account. Additionally, those field expressions already suggest that, though such conditions for CP may be met at one frequency, they will not be satisfied on a broad bandwidth. This is a consequence of the distinct frequency behaviors observed for $k_{y}^{10 / 01}$ in Section II, and the fact that $c^{10 / 01}$ may also be frequency dependent.

1. The starting point for the design is analytical. From the central operation frequency $\left(f_{0}=20 \mathrm{GHz}\right.$ in our case), the WG cross section is preliminary dimensioned to be squared $(a=b)$. Its size is chosen to achieve a desired pointing angle $\theta_{R A D}^{C P}=\theta_{R A D}^{10}=\theta_{R A D}^{01}$, by applying the well-known approximate relation with $\beta^{10}$ and $\beta^{01}$ found from a closed WG $\left(\theta_{R A D}^{C P}=35^{\circ}\right.$ results in $\left.a=b=9.16 \mathrm{~mm}\right)$ [11]. Next, the period between perforations is selected in order to avoid higher-order harmonics at $f_{0}(P=7 \mathrm{~mm})$ [4]. The size of the aperture $\left(L_{A}=136\right.$ $\mathrm{mm})$ is selected to attain high beam efficiency. It considers useful CP radiation coming only from the progressive LWs, and is defined as:

$$
\eta_{b}^{C P}=P_{R A D}\left(L_{A}\right) / P_{\text {in }}
$$

where $P_{\text {in }}$ is the incident power in the aperture, and $P_{R A D}\left(L_{A}\right)$ is the power leaked between $y=0$ and $y=L_{A}$. High values of $\eta_{b}^{C P}$ are thus attainable with high values of $\alpha^{10 / 01}$. As concluded in Section II, such scenario is possible when working at frequencies close and below the resonance of the crossed slots, when the aperture is highly transparent. The lengths of the crosses arms are thus initially chosen to be as long as possible, while still remaining below their first resonance. The value of $L^{01}$ is additionally limited by the period $P$.

2. This preliminary design is then simulated with a full-wave solver, being excited at its input and loaded at its end with ideal dualmode matched WG ports. A parametric analysis is carried out in order to tune the thickness of the metal walls $(t)$ and the widths of the crossed slots $\left(w^{10}\right.$ and $\left.w^{10}\right)$. The objective is to trade off high attainable value of $\eta_{b}^{C P}$ for manufacturability. 
3. The resulting design is modified afterwards through a coarse full-wave tuning of parameters $a, b, L^{10}$ and $L^{01}$, with the objective to reach $\alpha^{10} \approx \alpha^{01}$ and $\theta_{R A D}^{10} \approx \theta_{R A D}^{01}=\theta_{R A D}^{C P}$. Interpolation between simulated values is used to reach an approximate solution at a reasonable computational cost.

4. Finally, the previous design provides a robust starting point for a final full-wave optimization. The considered parameters are $a, b$, $L^{10}$ and $L^{01}$ and the objective is to reach the minimal AR and the desired $\theta_{R A D}^{C P}$.

\section{B. WG Feed Transition and Diffraction Mitigation}

Once the cross section of the perforated WG is defined, the appropriate WG feed transition can be designed in order to excite simultaneously both modes with equal power and phase. It should be noted that this feed component is very simple and does not implement any orthomode function. The feed has been designed to discard any direct radiation from the source, and discontinuities have been minimized to avoid diffraction. In this way, the leaky modes are the main source of radiation from this antenna. As shown in Fig. 1 (b), we consider a WG transition made of 5 consecutive sections. The first one is a standard WR42 rectangular WG section, allowing propagation of the fundamental $\mathrm{TE}_{10}$ mode. Then, a smooth taper of length $s_{2}$ transforms the rectangular cross section to a square one of size $a$ (the width of the perforated WG). The third part of length $s_{3}$ is inspired from [10] and enables dual-mode propagation. More precisely, the output port is rotated by $45^{\circ}$ relatively to the input one so that the fundamental mode is decomposed into the two degenerate $\mathrm{TE}_{10}$ and $\mathrm{TE}_{01}$ modes, with same power and phase. Next, a ridged waveguide of length $s_{4}$ is implemented to transform the $a \times a$ cross section to a $a \times b$ rectangular one. Tapered ridges allow for maintaining the same phase for the two output modes, despite the rectangular section. The last section, of length $s_{5}$, is a classical rectangular waveguide with a cross section $a \times b$. This feed transition has been optimized using full-wave simulations, its final dimensions are given in the caption of Fig. 1. The obtained performance is shown in Fig. 3. It is evidenced that the desired dual-mode output is obtained, with modes of almost equal powers and phases. The transition is also well matched with $\mathrm{S}_{11}<-15 \mathrm{~dB}$ over the frequency band of operation.

Additionally, it has been observed that diffraction occurs at the beginning of the perforated WG when connected to the feed transition, due to the exponential illumination of the aperture and its associated abrupt initial radiation [4]. Such phenomena may not only degrade the shape of the pattern but also the AR, since it does not affect equally the $\varphi$ and $\theta$ polarizations. A modulation of the four first crosses is applied and thus mitigates such deterioration. The leakage rates are therefore tuned along the $y$-axis, with the crosses dimensions found to reach the specified $\alpha^{10 / 01}$ while keeping the same beam efficiency $\eta_{b}^{C P}$ as the non-tapered design [8].

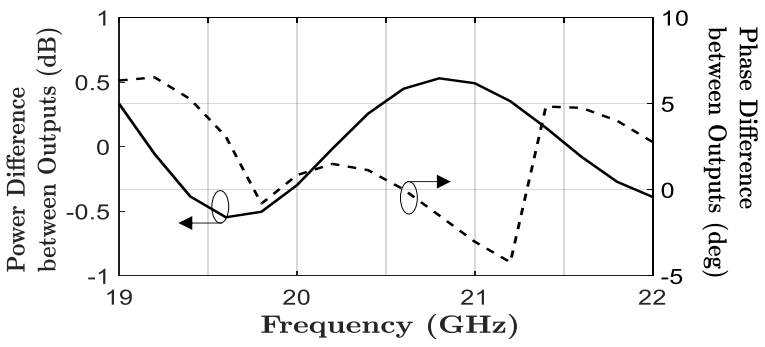

Fig. 3. Performance of the WG feed transition for dual-mode excitation.

Finally, the LWA end is loaded by a short circuit, as illustrated in Fig. 1. Such decision aims again at diffraction mitigation and keeping the LWA compact and short (to be compatible with small platforms). The energy remaining at the end of the antenna may radiate if it is left open. Non-negligible radiation from such discontinuity would affect differently $\theta$ and $\varphi$ polarizations [8], thus potentially spoiling the AR. Additionally, the short circuit allows reducing uncertainty in the antenna performance, since it isolates it from external interferences, and has a simple implementation. The power reflected backwards by the short circuit is radiated as crosspolarization in a direction opposite to the main beam. An alternative matched load can be employed in applications where such backward beam is not acceptable.

\section{Example of CP Performance}

The designed antenna is first simulated using an ideal input port exciting both degenerate modes (without the transition). Two different configurations are compared: one with a matched termination at the output and the other with a short circuit. The radiation pattern at $20 \mathrm{GHz}$ is exhibited in Fig. 4. Due to the high efficiency $\left(\eta_{b}^{C P}>85 \%\right)$, both terminations evidence very close performance in the main beam. The short circuit is then chosen, although a high backward cross-polarized beam is produced. The achieved pointing angle is $36.2^{\circ}$ with a maximum directivity of 16 $\mathrm{dBi}$. Low cross-polarization is observed in the main beam. The AR in the main beam is given in Fig. 5, and it is better than $3 \mathrm{~dB}$ on a 4 $\%$ bandwidth with a minimal value of $0.98 \mathrm{~dB}$ at $20.2 \mathrm{GHz}$.

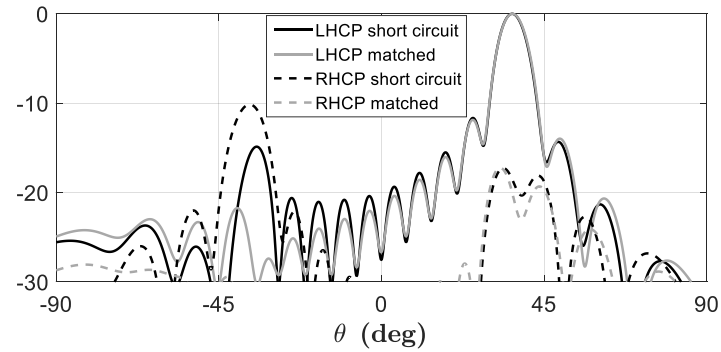

Fig. 4. Normalized radiation pattern $(\mathrm{dB})$ simulated at $20 \mathrm{GHz}$ in the $\varphi=\pi / 2$ plane. The perforated WG is excited at its input by an ideal dualmode port and ended by a short circuit or a matched load.

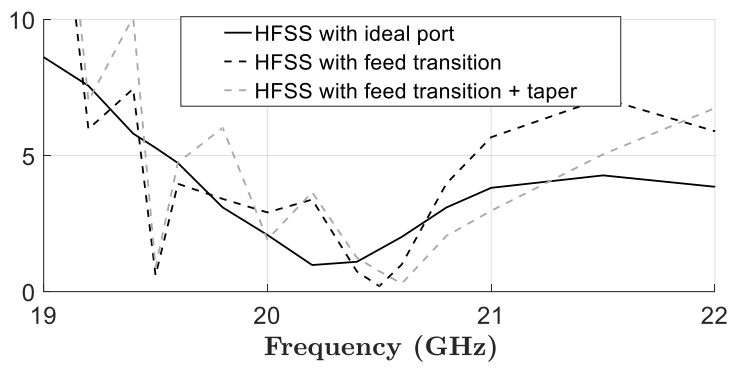

Fig. 5. AR (dB) in the main beam direction ( $\varphi=\pi / 2$ plane).

Fig. 5 plots the AR, considering three different configurations: with ideal feeding, with the transition designed in III. B, and finally, with application of the tapering procedure. The AR degradation associated to the use of the feed transition is evident in this figure, with a shift in the frequency of operation towards higher values. The taper allows for a slight improvement of the AR performance and bandwidth widening. The final design with crosses modulation provides LHCP polarization with AR of $0.3 \mathrm{~dB}$ at $20.6 \mathrm{GHz}$. The 3 $\mathrm{dB}$ AR bandwidth is $3.3 \%$ with a pointing angle at $39.3^{\circ}$ and a directivity of $15.9 \mathrm{dBi}$. The antenna is matched below - $10 \mathrm{~dB}$ over the full usable band, from $19 \mathrm{GHz}$ to $21.5 \mathrm{GHz}$. Further optimization of the final assembled antenna, or scaling of the metallic structure, are still possible actions to center the operation at $20 \mathrm{GHz}$. 


\section{Metal Additive Manufacturing}

The manufacturing technique selected for the prototyping of the CP LWA is the metal Additive Manufacturing (AM) process developed by the company SWISSto12. This process, which is presented here to the academic community for the first time, consists in two steps. The first step is the manufacturing of the component itself using Selective Laser Melting (SLM), while the second step is an electroless chemical treatment for roughness reduction followed by copper plating and silver passivation.

SLM can be used with different materials, but when using metals such as aluminum alloys, the raw manufactured part can already meet the typical mechanical and thermal requirements for space applications. This, as well as the fact that the raw part is nearly RFfunctional, is the main reason why SLM is typically the preferred AM process for WG hardware in the microwave industry [14], [15].

The main disadvantage of SLM is a poor surface roughness compared to common machining procedures. Additionally, the high porosity of the raw parts makes it difficult to plate the internal surfaces by standard methods. This often results in unacceptably high losses above Ku-band, even if a material with high bulk conductivity such as an aluminum alloy (AlSi10Mg) is used.

In order to overcome the poor surface roughness inherent to SLM, SWISSto12 has been active in developing a proprietary surface treatment process that leads towards significant RF loss reduction. This process consists of an electroless surface treatment that reduces the effective surface roughness and porosity, followed by copper plating for further effective conductivity improvement and silver passivation. The raw and plated equivalent conductivities of AM parts are below $0.5 \mathrm{e} 7 \mathrm{~S} / \mathrm{m}$ and $2 \mathrm{e} 7 \mathrm{~S} / \mathrm{m}$, respectively. These two values are estimated from the comparison between measurements and simulations of a large sets of waveguides from WR90 to WR28. It must be highlighted that the metal AM route developed by SWISSto12, and presented here, has been extensively tested through several qualification processes for flight programs between Ku-band and Q-band.

The SLM tolerances range from $50 \mu \mathrm{m}$ to $150 \mu \mathrm{m}$, depending on how the object is oriented inside of the 3D-printer. Generally, the best results are obtained when the structure does not present unsupported overhanging faces. If this is not the case, the structure is 3D-printed under certain angle, which leads to deterioration of the manufacturing tolerances as a consequence of the staircase effect [16]. Fig. 6 shows the CP LWA presented in this work. Two measurement jigs $\left(90^{\circ}\right.$ bend and twist) have also been produced using AM to facilitate the measurements in the anechoic chamber. To enable the best quality inside the dual-polarized waveguide, the antenna has been printed with the slots facing the printing platform. The cross-slots (see the shiny finish surrounding the slots in Fig. 6) and all the flanges have been re-worked after the SLM process, thus ensuring the best possible quality from this manufacturing run.
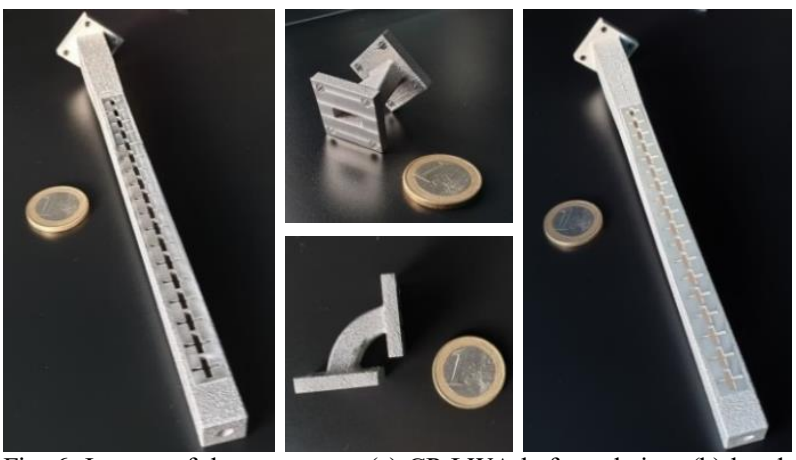

Fig. 6. Images of the prototypes (a) CP LWA before plating, (b) bend and twist for test (c) CP LWA after plating.

\section{Measured Performance and Discussion}

The CP LWA has been measured in a compact anechoic chamber between 19 and $22 \mathrm{GHz}$. According to Section IV, the following simulation curves have been updated compared to Fig. 4 and 5, taking into account additional metal thickness due to plating.

Fig. 7 shows the normalized radiation pattern at $20.5 \mathrm{GHz}$. The figure compares full-wave simulation and measurement. Both curves agree very well, with a pointing angle shifting of $0.9^{\circ}$. The measured cross-polarization has the expected magnitude, around $-22 \mathrm{~dB}$. There is also a significant backward beam, created by the presence of the short circuit at the antenna end. For applications where this beam is not allowed, a waveguide load can be used.

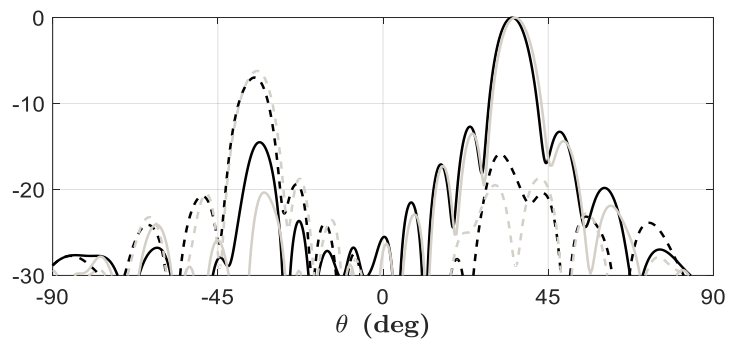

Fig. 7. Normalized radiation patterns (dB) in $\varphi=\pi / 2$ plane at $20.5 \mathrm{GHz}$. Continuous lines represent LHCP whereas dashed lines are RHCP. Grey lines are measured patterns while black curves are full-wave ones.
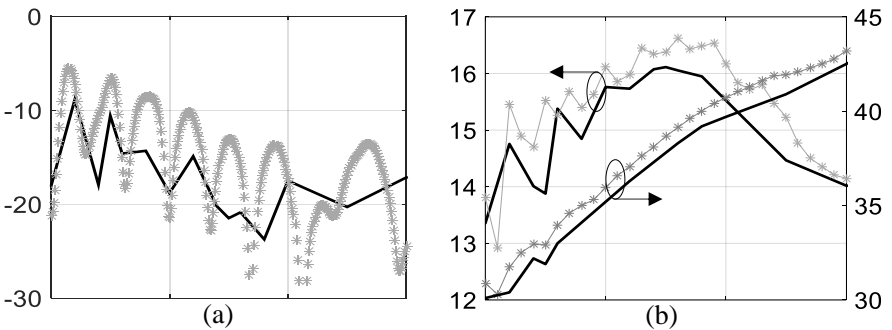

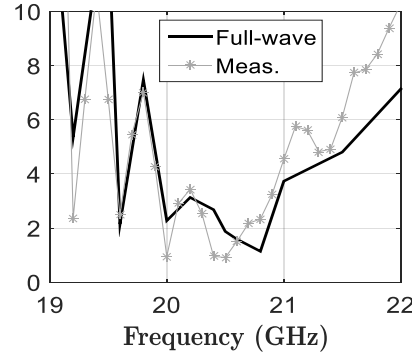

(c)

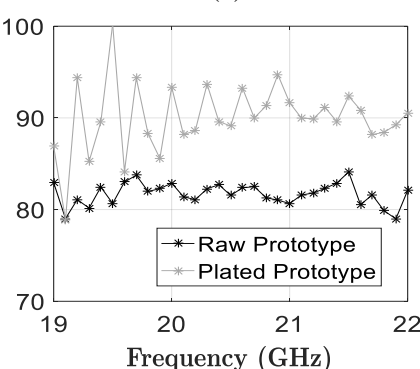

(d)
Fig. 8. Frequency performance of the prototype versus simulation, (a) matching (dB), (b) absolute gain ( $\mathrm{dBi}$ ) on the left and pointing angle (deg) on the right, (c) axial ratio $(\mathrm{dB})$ in the main beam direction for $\varphi=\pi / 2$, (d) radiation efficiency $(\%)$, raw versus plated prototype.

The reflection coefficient of the single LWA is shown in Fig. 8 (a). The measured value is higher than the simulated one. The differences are most probably caused by the fabrication tolerances and the precision of the measurement, done within a non-anechoic environment. The LWA is anyway well matched below $-10 \mathrm{~dB}$ above $20 \mathrm{GHz}$. The pointing angle at the operation frequency is $35.9^{\circ}$. The complete frequency behavior is shown in Fig. 8 (b), and it is in very good agreement with simulations (differences are always below 1 degree). The antenna absolute gain is shown in Fig. 8 (b). In this case, the difference must be attributed to the precision of the test setup. The cross-polarization discrimination is presented in Fig. 8 (c). The axial ratio is better than $3 \mathrm{~dB}$ within a $500 \mathrm{MHz}$ band centered at $20.55 \mathrm{GHz}$. This result, as well as the cross-polarization of Fig. 7, experimentally confirm that single-CP generation 
mechanism works well, and that the realization of the antenna is accurate. Finally, the radiation efficiency of the prototype is presented in Fig. 8 (d). It is worth reminding that the radiation efficiency differs from the beam efficiency, and takes into account the radiation of the LWs both in their forward path along $+y$ and backward one along $-y$. The conductivity enhancement achieved by the plating process represents a nearly $10 \%$-increasing of the radiation efficiency. The CP LWA shows a radiation efficiency of around $90 \%$. The device under test includes the bend and twist (which have not been plated) and the coaxial-to-WR42 setup excitation. Therefore, the CP LWA breadboard itself has an efficiency larger than $90 \%$.

The main features of this CP LWA are compared in TABLE I with other LWAs in the literature providing $\mathrm{CP}$ and based on hollow WGs. From this table it can be summarized that the proposed antenna provides better $\mathrm{CP}$ quality and bandwidth than previous proposals. Besides, it is the only one benefiting from monolithic prototyping, thus allowing for very high radiation efficiency, higher precision and robustness while avoiding the need for any assembly process. Furthermore, the LWA topology here proposed is the only one that allows a straightforward extension to dual-CP performance. This upgrade can be attained through AM easily by integrating an OMT in the feeding transition, which may excite the two $\mathrm{TE}_{01}$ and $\mathrm{TE}_{10}$ modes with either 0 or $\pi$ phase difference at the leaky guide input. It should be mentioned that such an antenna will have higher profile and less integration easiness compared to the existing reports on CP LWA employing dielectric substrates.

TABLE I

STATE-OF-THE-ART PERFORMANCE COMPARISON

\begin{tabular}{|c|c|c|c|}
\hline & $\begin{array}{c}{[6]} \\
21 \mathrm{GHz}\end{array}$ & $\begin{array}{c}{[7]} \\
\text { X-band }\end{array}$ & $\begin{array}{l}\text { This article } \\
20.5 \mathrm{GHz}\end{array}$ \\
\hline $3 \mathrm{~dB}$ AR bandwidth & $>0.5 \%$ & - & $>2.4 \%$ \\
\hline Absolute gain & $23.9 \mathrm{dBi}$ & $20 \mathrm{dBi}$ & $16.4 \mathrm{dBi}$ \\
\hline Pointing angle & $46^{\circ}$ & $40-45^{\circ}$ & $35.9^{\circ}$ \\
\hline $\begin{array}{c}\text { X-pol at } \\
\text { pointing direction }\end{array}$ & $<-19 \mathrm{~dB}$ & $<-13.5 \mathrm{~dB}$ & $<-22 \mathrm{~dB}$ \\
\hline $\begin{array}{l}\text { Radiation (antenna) } \\
\text { efficiencies }\end{array}$ & - - (70)\% & & $90(75) \%$ \\
\hline $\begin{array}{l}\text { Fabrication } \\
\text { technology }\end{array}$ & Subtractive & $\begin{array}{c}\text { Subtractive }+ \\
\text { attenuators / } \\
\text { phase shifters } \\
\text { assembly }\end{array}$ & $\begin{array}{c}\mathrm{AM} / \mathrm{SLM}+ \\
\text { treatments }\end{array}$ \\
\hline Extension dual-CP & No & Complex & Easy \\
\hline
\end{tabular}

\section{CONCLUSION}

A CP dual-mode LWA is presented here that is built only from metal. An original comparative dispersion study of the two leakymodes excited in the structure has been carried out for the first time, and evidences the different nature of both waves. Such differences justify that the combination of the radiation from both modes produces $\mathrm{CP}$ in a narrow band. A prototype operating at $20 \mathrm{GHz}$ is designed and built based on SLM additive manufacturing. For the first time, a plating post-processing step is conducted, proving its interest regarding the radiation efficiency improvement. The plated prototype shows a satisfactory measured performance with an AR of $0.92 \mathrm{~dB}$ at $20.5 \mathrm{GHz}$.

\section{ACKNOWLEDGMENTS}

The authors would like to thank Dr. L. Le Coq, from IETR, and L. Simon for their help in the experimental activities. This publication has been supported by the European Space Agency through an NPI grant, Rennes Metropole under Grant AIS 17C0481, the European Union through the European Regional Development Fund, and by Ministry of Higher Education and Research, Brittany \& Rennes Metropole, through the CPER Project SOPHIE / STIC \& Ondes.

\section{REFERENCES}

[1] R. E. Hodges, N. Chahat, D. J. Hoppe and J. D. Vacchione, "A Deployable High-Gain Antenna Bound for Mars: Developing a new folded-panel reflectarray for the first CubeSat mission to Mars.," in IEEE Antennas and Propagation Magazine, vol. 59, no. 2, pp. 3949, April 2017.

[2] R. Radhakrishnan et al. "Survey of Inter-Satellite Communication for Small Satellite Systems: Physical Layer to Network Layer View," in IEEE Communications Surveys \& Tutorials, vol. 18, no. 4, pp. 24422473, Fourthquarter 2016.

[3] N. Chahat, B. Cook, H. Lim and P. Estabrook, "All-Metal DualFrequency RHCP High-Gain Antenna for a Potential Europa Lander," in IEEE Transactions on Antennas and Propagation, vol. 66, no. 12, pp. 6791-6798, Dec. 2018.

[4] A. A. Oliner and D. R. Jackson, "Leaky-wave antennas," in Antenna Engineering Handbook, New York, NY, USA: McGraw-Hill, 2007.

[5] R. Lenormand, A. Hirsch, J. Almeida, A. Valero-Nogueira, J. I. Herranz-Herruzo and D. Renaud, "Compact switchable RHCP/LHCP mobile Ka-band antenna," 201215 International Symposium on Antenna Technology and Applied Electromagnetics, Toulouse, 2012.

[6] J. Hirokawa, M. Ando, N. Goto and M. Uematsu, "Low-Profile Mobile DBS Receiving System using a Single-Layer Slotted Leaky Waveguide Array," 1994 24th European Microwave Conference, Cannes, France, 1994, pp. 1553-1558.

[7] H. Hougardy and H. Shanks, "Arbitrarily polarized slot array," WESCON/58 Conference Record, Los Angeles, CA, USA, 1958, pp. 157-160.

[8] A. Dorlé et al., "Additive Manufacturing of Modulated Triple-Ridge Leaky-Wave Antenna," in IEEE Antennas and Wireless Propagation Letters, vol. 17, no. 11, pp. 2123-2127, Nov. 2018.

[9] M. García-Vigueras, E. Menargues, T. Debogovic, E. de Rijk and J. R. Mosig, "Cost-effective dual-polarized leaky-wave antennas enabled by three-dimensional printing," in IET Microwaves, Antennas \& Propagation, vol. 11, no. 14, pp. 1985-1991, Nov. 2017.

[10] J. M. Rebollar and J. de Frutos, "Dual-band compact square waveguide corrugated polarizer," IEEE Antennas and Propagation Society International Symposium., Orlando, FL.

[11] D. M. Pozar, Microwave Engineering, 2nd Edition, New York, Wiley, 1998.

[12] C. A. Balanis, Antenna Theory: Analysis and Design, 2nd ed. New York, NY, USA: Wiley, 1997.

[13] J. Liu, D. R. Jackson and Y. Long, "Modal Analysis of DielectricFilled Rectangular Waveguide With Transverse Slots," in IEEE Transactions on Antennas and Propagation, vol. 59, no. 9, pp. 31943203, Sept. 2011.

[14] O. A. Peverini et al., "Selective Laser Melting Manufacturing of Microwave Waveguide Devices," in Proceedings of the IEEE, vol. 105, no. 4, pp. 620-631, April 2017.

[15] P. Booth and E. V. Lluch, "Enhancing the Performance of Waveguide Filters Using Additive Manufacturing," in Proceedings of the IEEE, vol. 105, no. 4, pp. 613-619, April 2017.

[16] Charles, A.; Elkaseer, A.; Thijs, L.; Hagenmeyer, V.; Scholz, S. Effect of Process Parameters on the Generated Surface Roughness of DownFacing Surfaces in Selective Laser Melting. Appl. Sci. 2019, 9, 1256. 